\title{
A rare case of intracranial malignant melanoma with an unusual presentation
}

\author{
Sandeep B V1', Suniti Kumar Saha², Manpreet Singh Banga', Partha Ghosh ${ }^{1}$ \\ ${ }^{1}$ Post Doctoral Trainee, ${ }^{2}$ Professor and Head, Department of Neurosurgery, Nilratan Sircar Medical College and \\ Hospital, Kolkata, West Bengal, India
}

Among the common skin cancers, melanoma is the most lethal. Although, it comprises only $3 \%$ of all skin cancers diagnosed, it accounts for about $75 \%$ of all skin cancer-related deaths. Melanoma is a relatively uncommon skin cancer in geographical locations like India. Its highest incidence is seen in sixth decade. Head and neck melanomas constitute approximately $17 \%$ of all cutaneous melanomas. We present a 15 year old male patient who presented with a intracranial melanoma with osteolytic skull lesion.

\section{Access this article online}

Website:

http://nepjol.info/index.php/AJMS

DOI: 10.3126/ajms.v7i6.15201

E-ISSN: 2091-0576

P-ISSN: 2467-9100

Key words: Melanoma, Osteolytic skull lesions, Intracranial melanoma

\section{INTRODUCTION}

Among the common skin cancers, melanoma is the most lethal. Although, it comprises only $3 \%$ of all skin cancers diagnosed, it accounts for about $75 \%$ of all skin cancer-related deaths. The incidence and mortality rates are decreasing or leveling off in the younger population, but are still increasing in the older age groups. The rate of increasing incidence varies geographically with "high incidence regions" like Australia, "moderate incidence regions" like Canada and USA, and "low incidence regions" like india and scotland. ${ }^{1}$ Osseous metastases of malignant melanoma are rare among Asians. ${ }^{2}$

\section{CASE REPORT}

A 15 year old male patient presented to our OPD with complaints of painless swelling in the posterior aspect of scalp since 1 year which was insidious in onset gradually progressive in nature. Patient gave a history of hyper pigmented patch over that area since birth. On examination there was a 15 x $20 \mathrm{~cm}$ non tender swelling in the parieto occipital region which was firm in consistency fixed to underlying bone. CT brain was done which showed heterogeneously contrast enhancing mass with underlying bony defect in parieto occipital region (Figure 1). MRI brain was done. T1 weighted images suggested heterogeneous mass which was isointense with few areas of hyper intensity with intracranial extension. T 2 weighted images suggested heterogeneous mass which was isointense with areas of hyper intensity. On contrast administration there was heterogeneous enhancement of contrast (Figure 2). Chest X ray was normal. Ultrasound abdomen was suggestive multiple metastatic lesions in the liver. Patient was operated, intra operatively. The mass was blackish in color moderately vascular with intra cranial extension with eroded bony margins was seen (Figure 3). Debulking of the mass was done followed by covering of exposed area with rotation flap. Histopathological examination was suggestive of malignant melanoma (Figure 4). Patient was referred to chemo radiotherapy department for further evaluation and management.

\section{DISCUSSION}

Melanoma is a relatively uncommon skin cancer in geographical locations like India. Its highest incidence is seen in sixth decade. Presentation differs between genders. The commonest site in males is the trunk followed by head and neck, whereas in females it is the lower limb. Males usually present at an older age and with a later stage of disease. Melanoma in children and adolescents is rare and remains understudied. It accounts for less than 3\% 


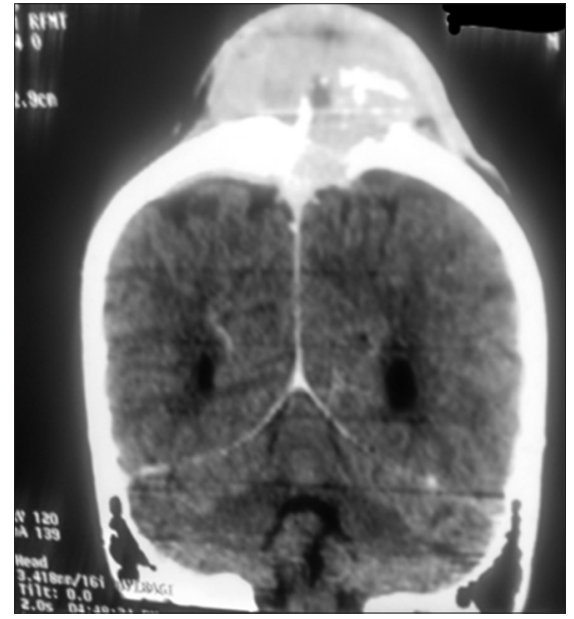

Figure 1: CT scan brain with contrast (coronal view)

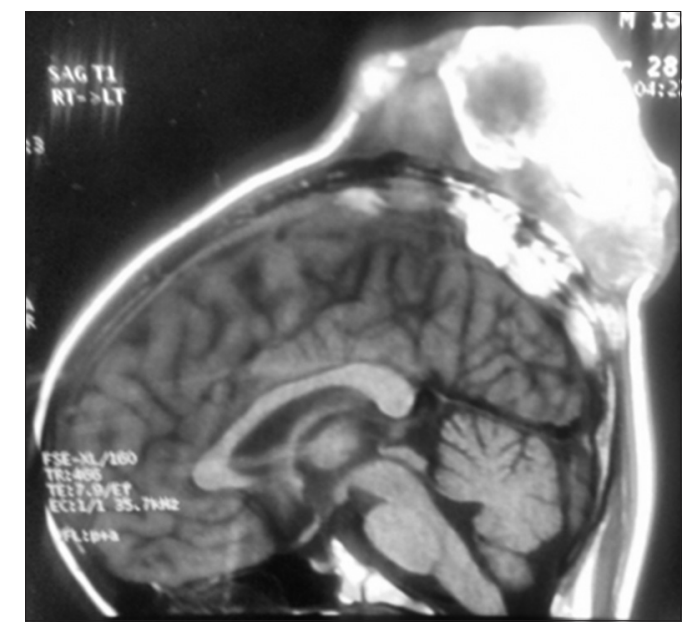

Figure 2: MRI T1 sagital view

of all childhood neoplasms. ${ }^{1}$ Head and neck melanomas constitute approximately $17 \%$ of all cutaneous melanomas. ${ }^{3}$ In our case, Patient is a 15 year old male with malignant melanoma over the scalp.

Most melanomas have two phases of growth, an initial radial (horizontal) and a later vertical growth phase. The radial phase is not associated with spread, but once vertical phase supervenes, it gives rise to cell population that can metastasize. The rate of distant spread is closely related to the number of involved nodes, whereas the risk of regional (in-basin) recurrence is related to the extracapsular extension of the nodal disease. ${ }^{4}$ Metastasis occur to draining lymph nodes, lung, liver and bone. Common sites for osseous metastases of melanoma include the vertebrae, skull, pelvis, thoracic cage and proximal parts of the femur. ${ }^{5}$ In our case the melanoma had involvement of the underlying bone, manifesting as an osteolytic lesion over parieto occipital bone along with intracranial extension. Metastatic lesions In liver were also seen.

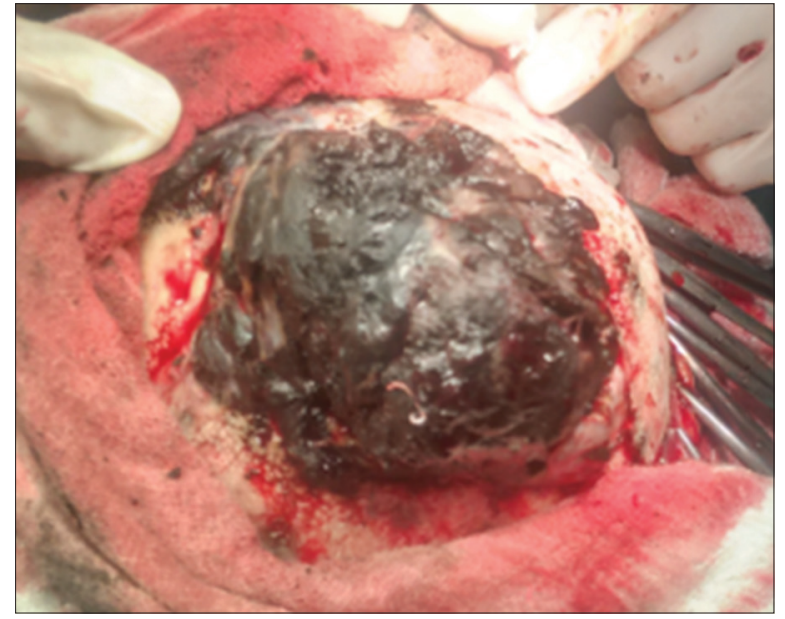

Figure 3: Intraoperative picture

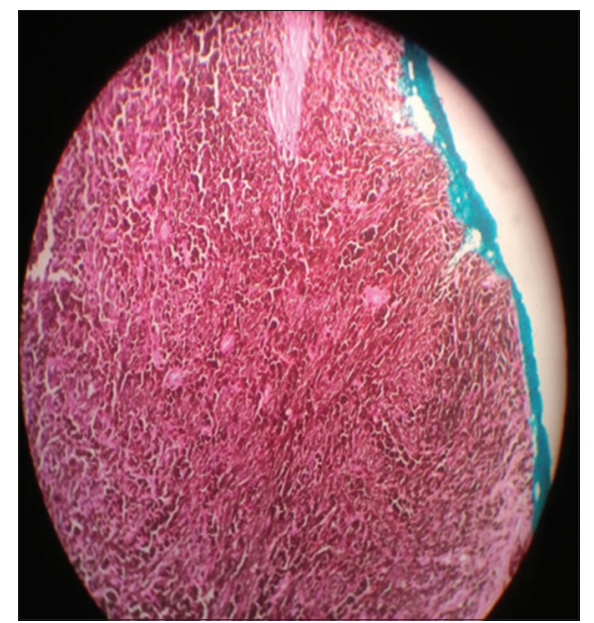

Figure 4: Histopathological picture

We found a similar presentation as our case on extensive search of articles, where a 23 year old male patient presented with osteolytic skull lesions in frontal skull bone which on excision reported as malignant melanoma with unknown primary. ${ }^{6}$ In our case there was osteolytic skull lesion over parieto occipital region.

Whereas the overall prognosis of an earlier diagnosed thin tumor is excellent, once metastasized it becomes an incurable disease with high mortality. Regarding the prognostic importance of various factors, the anatomical site of stage IV tumors correlates with survival, with metastasis to brain and visceral sites having the worst prognosis. $^{1}$

Treatment of melanoma involves multimodality approach involving surgery, radiotherapy, chemotherapy, and immunotherapy. Surgery includes resection of the primary, lymph node dissection, sentinel lymph node biopsy, and resection of metastases in selected cases. ${ }^{1}$ 
In selected Stage IV melanoma patients, aggressive surgical approach has been reported since non-surgical treatment of Stage IV melanoma has been beneficial in only few selected cases. ${ }^{7}$ In our case debulking of the tumour was done and patient was referred to chemo radiotherapy department for further management.

\section{CONCLUSION}

The possibility of metastatic melanoma should be considered as the differential diagnosis of any lytic bony lesion in the skull even in a young adult.

\section{REFERENCES}

1. Sharma K, Mohanti BK and Rath GK. Malignant melanoma: A retrospective series from a regional cancer center in India.
Journal of Cancer Research and Therapeutics 2009; 5:173-180.

2. Lange JR, Sharfman $\mathrm{WH}$, Alani RM and Balch CM. Melanoma. Clinical Oncology, Churchill Livingstone 2004; 1561-1583.

3. Fisher SR. Cutaneous malignant melanoma of the head and neck. Laryngoscope 1989; 99:822-836.

4. Ballo MT, Ross MI, Cormier JN, Myers JN, Lee JE, Gershenwald JE, et al. Combined modality therapy for patients with regional nodal metastases from melanoma. International Journal of Radiation Oncology Biology Physics 2006; 64:106-113.

5. Kuo- yuan Huang, Chrong- Reen Wang and Rong -SenYang. Rare clinical experiences for surgical treatment of melanoma with osseous metastases in Taiwan.BioMedCentral Musculoskeletal Disorders 2007; 8: 70.

6. Shenoy AS, Desai HM, Kavishwar VS and Savant HV. Metastatic malignant melanoma in a young adult with unknown primary. Indian Journal of Cancer 2015; 52:446-447.

7. Komorowski AL, Wysocki WM and White RL. Surgical management of solitary metastatic melanoma.Acta Chirurgica Belgica 2009; 109:155-158.

\footnotetext{
Authors Contribution:

SBV - Principle author - reviewed the literature, manuscript preparation and critical revision of the manuscript; SKS - Operated the patient, reviewed the literature, manuscript preparation; MSB - Literature search, prepared first draft of manuscript and critical revision of the manuscript; PG - Collected data and review of study, literature search.
}

Source of Support: Nil. Conflict of Interest: None declared. 